\title{
CORRELATING DESIGN PERFORMANCE TO EEG ACTIVATION: EARLY EVIDENCE FROM EXPERIMENTAL DATA
}

\author{
Li, Shumin; \\ Becattini, Niccolò; \\ Cascini, Gaetano \\ Politecnico di Milano
}

\begin{abstract}
This paper presents an EEG (Electroencephalography) study that explores correlations between the neurophysiological activations, the nature of the design task and its outputs. We propose an experimental protocol that covers several design-related tasks: including fundamental activities (e.g. idea generation and problem-solving) as well as more comprehensive task requiring the complex higher-level reasoning of designing. We clustered the collected data according to the characteristics of the design outcome and measured EEG alpha band activation during elementary and higher-level design task, whereas just the former yielded statistically significant different behaviour in the left frontal and occipital area. We also found a significant correlation between the ratings for elementary sketching task outcomes and EEG activation at the higher-level design task. These results suggested that EEG activation enables distinguishing groups according to their performance only for elementary tasks. However, this also suggests a potential application of EEG data on the elementary tasks to distinguish the designers' brain response during higher-level of design task.
\end{abstract}

Keywords: Electroencephalography, Neurocognition, Human behaviour in design, Creativity, Design cognition

\section{Contact:}

Li, Shumin

Politecnico di Milano

Mechanical Engineering

Italy

shumin.li@polimi.it 


\section{INTRODUCTION}

Neurophysiological measures are increasingly adopted in design cognition studies (Abraham, 2018; Benedek et al., 2018) to overcome the limitations of traditional approaches based on observation, interviews and questionnaires, which highly depend on the observer's interpretation. The neurophysiological techniques allow obtaining quantitative data measured directly from subjects' brain along with design activities, thereby enable to acquire more objective data to analyse design cognition. There are various kinds of apparatus for measuring different neurophysiological signals. While each of these techniques provides a different indication of brain activities, they also come along with different limitations. Unlike in clinical research, these limitations highly affect the development of the experimental protocol in design cognition studies. Functional Magnetic Resonance Imaging (fMRI) requires the subjects to stay in the scanning tunnel and react to stimuli by hand or by verbalizing the experience after data acquisition. Near-Infrared Spectroscopy (NIRS) is limited to frontal regions and surface analysis, with interpretation challenges related to multiple sources of the vascular signal.

Electroencephalography (EEG) was originally used in clinical research, especially to diagnose brain dysfunction and study sleeping. Soon enough, thanks to the availability of low-cost devices, the simplicity of installation and well-developed basic analysis tools, it started to play a role in psychological studies on diverse kinds of behaviour (Arden et al., 2010). EEG measurements are spreading in design cognition studies as well, but they are still at an exploratory stage (Göker, 1997; Nguyen and Zeng, 2010; Vieira et al., 2020). These efforts revealed some correlation between brain activation and design behaviour; however, there are no established experimental procedures yet and results are not consolidated. As a matter of fact, the EEG signals can be biased by multiple sources of noise and despite different methods of data processing that have been developed, their interpretation requires a good deal of art (Jiang et al., 2019).

Considering the different behaviour of designers with different background in performing design activities and the lack of studies with a specific focus on engineering design, the current research project targets the study of neurocognition in engineering design tasks starting from subjects with an engineering background. In this perspective, the research project aims at establishing a reference protocol suitable to identify the relationships between neurophysiological activations, task nature and task outputs.

This paper first briefly introduces what has been achieved in design cognition studies through neurophysiological data. Then it presents the experimental protocol of the current research work and the adopted data treatment pipeline. After that, the preliminary results from the data collected within the first experimental campaign are illustrated. The discussion based on these results closes the paper.

\section{THE STATE OF THE ART}

\subsection{Design cognition studies with neurophysiological data}

In design research, human behaviour was originally studied using observation through protocol analysis, then sometimes correlating the behaviour to the design outcome e.g., through the assessment by experts. Questionnaires and interviews shed a light on some mechanisms to achieve a good quality of design, but still can't reflect the ongoing brain activity along the design process, so that the correlation with the time dimension is still lacking. This requires more objective data, such as neurophysiological ones, to describe the design cognition while performing the design activity, as this could be one of the keys for the exploration of design behaviour. Diagnostic studies about brain conditions are tailored for the identification of out-of-the-norm conditions, therefore those experimental protocols are not suitable to investigate differences between healthy subjects with no brain dysfunctions. Starting from elementary design-related activities, different techniques are under investigation in design studies.

Favoured by its high spatial resolution, fMRI was used for mapping the cognitive activities across the whole brain. For instance, Knyazev (2007) explored neural networks involved in artistic creativity, asking the subjects to imagine a new design for a pen. He observed the degree of dominance of the right over the left prefrontal cortex among experts and novices and found differences in brain activation also within each group. Alexiou, et al. (2011) investigated brain activities of design experienced participants with fMRI. They observed different patterns of functional interactions between brain regions along with 
the execution of design and problem-solving tasks, suggesting that design and problem-solving involve distinct cognitive functions associated with distinct brain networks. Chávez, et al. (2004) used SinglePhoton Emission Computed Tomography (SPECT) to investigate figural and verbal creativity with the Torrance Test for Creative Thinking (TTCT, (Torrance, 1968)) and found correlations with cerebral blood flow at different Brodmann Areas. While fMRI and SPECT techniques limit the movements of the subject, NIRS and EEG instruments allow the subject to stand up or sit down and designing in a close-toreal environment. By NIRS, Gibson, et al. (2009) studied creativity with the Remote Associates Test (Mednick, 1968). The data showed greater bilateral frontal activity in musicians during divergent thinking compared with non-musicians. Similarly, Martindale and Hines (1975) tested creative behaviour through an early EEG exploration and they discovered that groups with higher and lower creativity scores also had different brain behaviour.

Among different types of neurophysiological data, EEG signal, which describes the voltage fluctuations within the neurons of the brain, can provide an excellent temporal resolution. This allows exploring the brain activation at the level of a millisecond (Kounios and Beeman, 2014), but also through longer time frames from minutes to the whole time required for a design task (Vieira et al., 2020).

\subsection{EEG studies in psychology research}

EEG measurement is commonly used in cognitive psychology studies for exploring the reaction to different stimuli through brain activation, e.g. music vs. text (Bhattacharya and Petsche, 2005), image vs. text (Appelbaum et al., 2010). Another research topic deals with the different brain functional areas involved within an elementary task, e.g. in memory tasks (Klimesch et al., 1999), or selective attention (Foxe and Snyder, 2011) and with measuring the mental load (Smith et al., 2001). Differences in terms of EEG activation appear between groups characterized by different gender (Razumnikova, 2004), years of experience (Fink et al., 2009) and age (Privodnova et al., 2017). The reaction to the trigger usually appears quickly after the stimulation and lasts within a few seconds, so that the experiments usually consist of several repetitions of one single request. The classic task designed by Guilford (1967), namely Alternative Uses Task (AU or AUT), has been commonly used as a reference test to collect diverse kind of neurophysiological data and check for correlates with divergent thinking. The test asks the participant to think of as many uses as possible for a simple object so that it allows each subject to repeat the test with different objects by thinking aloud. Torrance's TTCT is a wellestablished test for assessing creativity. This includes both verbal and non-verbal tests, allowing the subject to generate ideas by sketching in silence.

The EEG signal is by nature sensitive to many sources of electromagnetic perturbation, which include the nerve's stimulation of the muscles, as well as eye movements. These perturbations, namely "artefacts", should be avoided for a good quality of EEG signal acquisition and therefore tasks of short duration are preferable as they limit the perturbation due to eye movements.

Both AU and TTCT tests provide methods to assess creativity, but it should be noted that designing substantially differs from creativity tasks as commonly referred to in psychological literature. Within a design activity, one might carry on multiple cognitive functions, in a loop between divergent and convergent thinking. When shifting from creativity study to design research with EEG-based data, the nature of the task complicates the signal composition by involving more body movements. For instance, sketching involves neck and arm movements, the verbalization of ideas with details involves facial expression, and limiting the eye movements would make the behaviour unnatural. Therefore, the development of an experimental protocol to study design behaviour with EEG, requires establishing a careful signal processing pipeline that is able of removing different types of artefacts without constraining the subjects' movements excessively.

Studies across different research fields of the waveform of EEG discovered that brain oscillations exist over a number of functional domains (motor, visual...), with different frequency rhythms associated with each domain (Knyazev, 2007). Among these, the alpha oscillations $(7-13 \mathrm{~Hz})$ are the most salient EEG band, and thus they were studied the most. Synchronization and localization of the creative cognition within frontal, posterior, or lateralized hemispheric cortices are often claimed, e.g. Arden, et al. (2010). In addition, individual differences in brain structure and volumes, condition of hair and scalp, mental fatigue states can induce a different level of activation in terms of the absolute power of EEG (Jackson and Bolger, 2014). The wide variety of conditions that characterize the way the brain behaves also requires the selection of a baseline to calculate event-related power (ERP) in EEG data analysis. 


\subsection{The goal of the current research work}

This paper presents the preliminary results of a larger experiment that aims to explore the relationships between neurophysiological activations, the nature of the design task and its outputs. The analysis reported in this paper focuses on novice engineering students' neurophysiological activations collected with an EEG headset while addressing a sequence of tasks of different nature also including a typical design task. It explores the degree of activation during the execution of those different tasks. It also analyzes the opportunities that emerge from clustering subjects according to their design outcome or EEG activations and the coherence between these different approaches. Overall, it aims at understanding how EEG data can inform the analysis of design cognition processes.

\section{METHOD}

\subsection{Experimental protocol}

The development of the current experimental protocol leveraged early evidence from a set of pilot tests that aimed at finding the best trade-off between the quality of the EEG signal and real-like working condition for a design session carried out individually.

Before each experiment starts, the subject receives information about the EEG device used for data acquisition, an informed consent form to sign and the data treatment policy, as approved by the ethical committee of the authors' research institution. Then, the subject sits in front of a computer. The experiment supervisor properly places the headset on the subject's scalp to ensure good contact quality.

The subject then proceeds through five tasks (Figure 1), each preceded by a Wisconsin Card Sorting Test (WCST, (Heaton et al., 1993)) that lasts for 36 seconds (at most). In WSCT, the subject must select the only card out of four that shares the same feature (e.g. shape, colour...) with a randomly displayed card. Due to the low cognitive workload it requires, it allows the subject to relax and have a basic cognitive activation before each task. This also serves as a baseline for the estimation of taskrelated activation and, due to the duration of the experiment (approximately 30 minutes), to clearly separate cognitive activities between two subsequent tasks.

Each participant starts with the same warm-up task (n-back, (Kirchner, 1958)) to get familiar with the laboratory setting and the keyboard operation (duration: 20 seconds). The subject sees a series of letters as stimuli and, from the third letter on, it also has to type the letter seen two steps before. This also distracts the subject from the presence of the headset before it addresses the next tasks. Then, the protocol has three other tasks that entail more fundamental thinking and one that requires higher-order thinking, which is more typical of design activities. In general terms, the latter requires the subject to generate a design solution that satisfies a set of requirements with the help of a morphological table (DwMT); the former three include a problem-solving task where the subject has to exploit her/his knowledge in physics (PS), the AU task and the TTCT (in its figural variant). The last two tasks aim at confirming the validity of data acquisition with reference to existing studies in the literature, also considering the differences among the devices used in experiments carried out by other scholars.

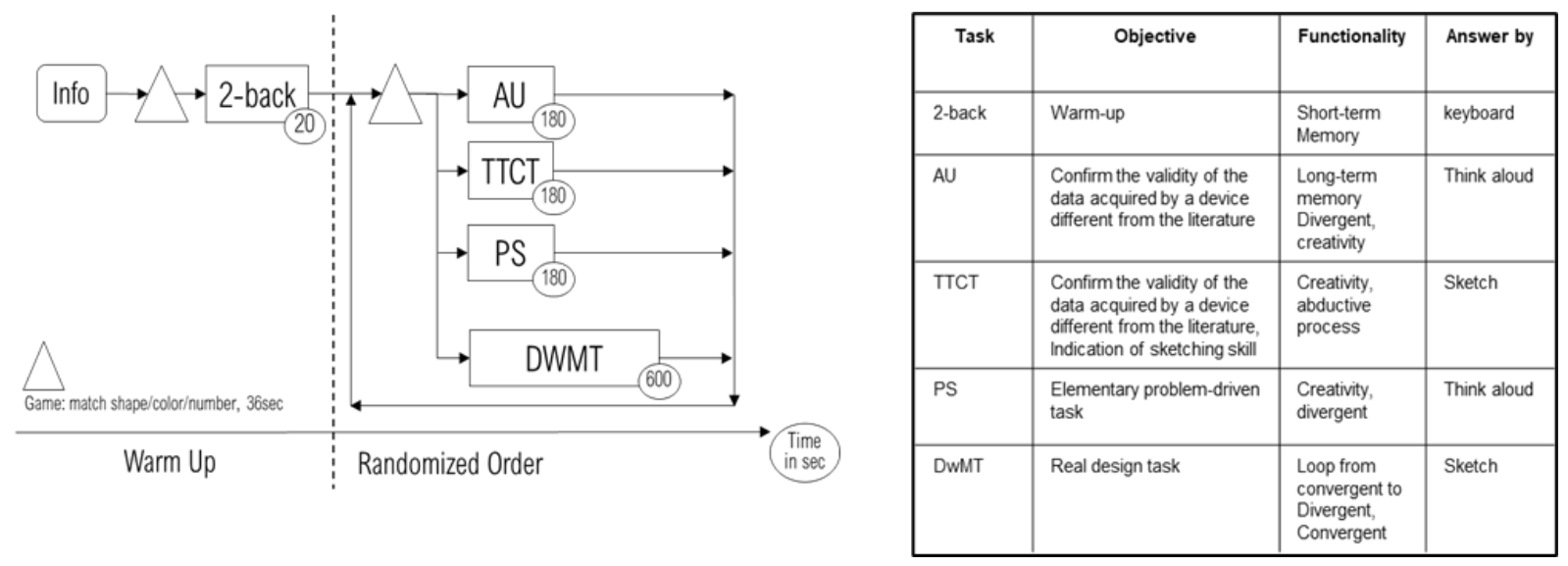

Figure 1. Experimental protocol. AU-Alternative Uses test, TTCT - Torrance Tests of Creative Thinking, PS - problem solving, DwMT - Design with Morphological Table.

Both DwMT and PS enabled the study to challenge the subjects (novice engineering designers) and to differentiate their brain activities as these two tasks entail different cognitive processes. The DwMT 
(duration: 10 minutes) asks the subject to design an amphibious bike; the subject is provided with a morphological table, which includes several options per product feature/function (e.g. propulsion system, number of allowed passengers...).

The PS task (duration: 3 minutes) requires the subject to generate and verbalize as many solutions as possible to empty a glass of water placed on top of a large heavy table without touching it.

The TTCT-figural (duration: 3 minutes) requires the subject to complete a figure, initially composed just of two vertical lines, by sketching on top of them and assign the sketch a name that clarifies its meaning. The subject can repeat this within the given time as many times as one wants. Besides, the TTCT allows the evaluation of the subject's sketching skills and, therefore, data triangulation with DwMT results. Eventually, during the AU task (duration: 3 minutes) the subject has to verbalize alternative uses for a cork. This also allows potential triangulation for the presence of artefacts during the PS task.

These 4 tasks are randomly ordered, so that any potential bias due to cognitive fatigue decreases its effect across multiple-subjects aggregated data, to check for general design/brain activation correlations.

All the experiment sessions are audio/video recorded to facilitate data-processing activities, such as the detection of body movements triggering EEG artefacts and the distinction of different stages within the same task (e.g. reading the assignment, sketching/drafting, analysing and selecting options from the morphological table). Especially with reference to DwMT, which lasts for a longer time and requires the subject to carry out several activities, different cognitive functionalities might be involved in sub-stages of the same task. Therefore, different kinds of brain activations are likely to characterize these sub-stages, thus defining different epochs to be analysed separately.

The experimental process is programmed by Psychopy 3 (Peirce et al., 2019), whose log file enables the synchronization of the whole dataset (EEG, video recording and keyboard operation). The subject receives information about the task and its execution through the screen of the computer and can ask clarification questions before starting the task. There is no time limit for reading the instructions. The subject also receives on-screen feedback for the time left or, just for tasks requiring sketching, sound notifications.

\subsection{Criteria for the assessment of task outcomes}

As each task returns different outcomes, they require different metrics. This assessment allows the clustering of subjects according to the results they had after each task.

The set of figures sketched during TTCT are measured according to fluency (estimator: number of completed figures), originality (estimator: number of ideas that are least proposed within the cohort) and variety (estimator: number of categories of ideas). Similarly, the PS task returns a set of generated and verbalized ideas that are also measured in terms of fluency (estimator: number of solutions) and variety (estimator: number of physical effects in the subject's solution set). The DwMT task returns a sketch with annotation about the final design for the amphibious bike. The metrics used for this unique idea are the level of detail/clarity and viability of the solution, both assessed with a 4-level Likert scale. The reliability of these assessments is obtained through consensus among two experts. The AU is the least design-related task and there is no assessment for its outcomes, as it was just used to confirm the validity of EEG data with reference to other studies in the literature.

\subsection{EEG Data Processing Pipeline}

EEG data collection should be carried out with a sampling frequency of a minimum of $128 \mathrm{~Hz}$, coherently with the Nyquist-Shannon sampling theorem, to allow studying all the sub frequency bands that are potentially relevant for neurocognition (from the delta-band [0.1 - 4] $\mathrm{Hz}$ to the lower gammaband $[24-40] \mathrm{Hz})$. However, having noticed that the delta band $(0.1-4 \mathrm{~Hz})$ is highly contaminated by noise and muscular artefacts, it is no longer considered in the current research. Figure 2 describes the data processing pipeline as a flow chart. Initially, an Infinite Impulse Response (IIR) filter removes the DC offset that is typical of the headset used for the experiment. Then, a bandpass filter cut-off frequencies outside the $4-45 \mathrm{~Hz}$ range, coherently with what has been stated above. The third step addresses the removal of muscular artefacts via the blind source separation (BSS) technique, based on the canonical correlation analysis (CCA) (Borga and Knutsson, 2001; De Clercq et al., 2006). The BSSCCA algorithm is adapted to remove the short Electromyography (EMG) bursts due to the articulation of spoken language and further attenuate the contamination of EEG data by muscular artefacts recordings (Vos et al., 2010). The fine-tuning of BSSCCA filtering parameters (window length 
2.5 seconds, window shift $1.2 \mathrm{~s}$ and removal of the 4 least correlated components) was done through the analysis of EEG data collected during pilot tests and intentionally contaminated with muscular artefacts taking place during the above 5 tasks (e.g. hand movements, neck rotations and eye movements). An additional stage for the removal of segments with muscular artefacts is possible through the analysis of audio and video recordings, as well as outliers in terms of moving average exceeding the threshold of $200 \mu \mathrm{V}$, above which the amplitude of the contamination is too high to be removed by the previous steps. Eventually, a set of bandpass filters divides the data into sub-frequency bands. Among them, this paper focuses on the alpha band $(7-13 \mathrm{~Hz})$. The normalization of data was done according to the following formula for Task Related Power (TRP) estimation, one estimator per each of the five tasks.

$$
T R P_{i j}=\frac{P O W_{i j}(\text { task })}{P O W_{i j}(r e f)}
$$

Where POW is the average power of the electrode $i$, from subject $j$ across one task (numerator) or across the reference WCST (denominator). The use of the logarithmic scale for TRP facilitates the visualization of brain activation. In such a case, a positive TRP indicates that the subject had stronger brain activation during the task than the reference WCST and vice versa.

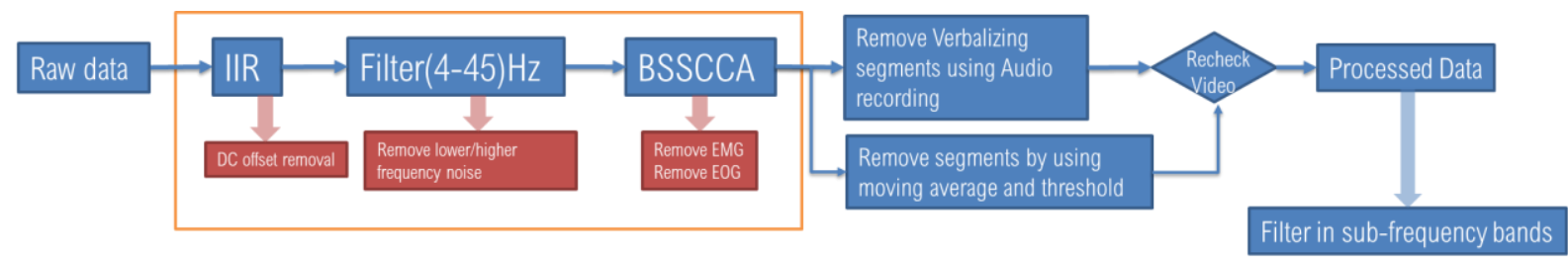

Figure 2. Signal processing pipeline

The whole data processing pipeline leverages an original Matlab script and the EEGLab toolbox (Delorme and Makeig, 2004).

\subsection{Criteria for the statistical analysis of differences between clusters of subjects}

The detection of differences in EEG data between individuals is typical of medical diagnostics to check whether an individual is ill or not. The search for correlates between EEG activation and design activities, in turn, requires a higher degree of generalization in order to generate more universally valid conclusions. This means that the analysis should involve clusters of subjects that show similar behaviour with each other. After filtering for outliers, the procedure to cluster subjects into homogeneous subsets can be either based on their brain activation level calculated by the unweighted average distance using Matlab linkage function or based on the assessment of their task outcomes. Then, the comparison between the two (or more) clusters for the search of correlates should be carried out with the dataset that was not used for clustering. E.g. if clusters of subjects are created according to EEG data, the search for correlates between brain activation and task outcome should be done by checking if the two clusters are also statistically different according to the metrics used to assess their task outcomes, and vice versa. Both the clustering approaches appear to be equally valid for this exploratory study.

The statistical testing of hypotheses uses parametric tests (two-sample t-test for the mean, homo/heteroskedastic, depending on the dispersion of data in the two clusters) if the data in clusters are normally distributed. On the other hand, non-parametric tests should be used for hypothesis testing and, in such a case; the two clusters are compared against each other with the Kolmogorov-Smirnov test.

\section{RESULTS}

\subsection{Participants and EEG acquisition device characteristics}

A subset of already acquired EEG data for homogenously characterized subjects can be considered for this exploratory study. This subset comprises 19 undergraduate engineering students, all of them were male, right-handed and novice engineers (experience $\mathrm{M}=1.14, \mathrm{SD}=0.51)$, aged 18-21 $(\mathrm{M}=18.96$, $\mathrm{SD}=0.73$ ).

The headset used for EEG data collection is Emotiv EPOC+. It has a pre-mounted frame with 14 electrodes (AF3/4, F7/8, F3/4, FC5/6, T7/8, P7/8, O1/2), sampling at $128 \mathrm{~Hz}$. The raw data is accessible to run signal processing according to the needs. 


\subsection{Statistical Analysis and Clustering Approach}

\subsubsection{Clustering according to the score based on the assessment of task outcomes}

For each task, the subjects were firstly clustered into two groups according to the scores they got for their task outcomes (sect. 3.2). Group A is for top-score subjects, group B is for the residuals. In such a way, clusters of subjects possibly have a different composition for each task. For TTCT group A and B have 10 and 9 subjects respectively. Because of an EEG activation beyond the $95 \%$ confidence interval, 5 subjects have to be removed before clustering them for the PS task according to their outcomes. Then, group A and B have 6 and 8 subjects in each. For the same reason, 2 outliers were excluded before clustering subjects for DwMT. Here group A and B respectively have 6 and 11 subjects. The statistical analysis thereby was performed separately for each task. None of the datasets within each cluster is distributed normally. Thereby, Table 1 presents the $p$-values for the TTCT and PS tasks, according to a two-sample Kolmogorov-Smirnov test based on the EEG data, under the null hypothesis that there is no difference between the clusters. For TTCT, the two groups present statistically significant differences for the F7 $(\mathrm{p}=0,047)$ and FC5 $(\mathrm{p}=0,002)$ channels. For task PS, only the channel P7 ( $\mathrm{p}=0,034)$ shows statistically significant differences between the groups. The full task analysis for DwMT returned no statistically significant differences at all.

\begin{tabular}{|r|c|c|c|c|c|c|c|}
\hline Left Hemisphere & AF3 & F7 & F3 & FC5 & T7 & P7 & O1 \\
\hline TTCT & 0.603 & $\mathbf{0 . 0 4 7}$ & 0.112 & $\mathbf{0 . 0 0 2}$ & 0.314 & 0.148 & 0.352 \\
\hline PS & 0.603 & 0.603 & 0.603 & 0.603 & 0.278 & $\mathbf{0 . 0 3 4}$ & 0.789 \\
\hline Right Hemisphere & AF4 & F8 & F4 & FC6 & T8 & P8 & O2 \\
\hline TTCT & 0.112 & 0.438 & 0.603 & 0.166 & 0.176 & 0.375 & 0.249 \\
\hline PS & 0.278 & 0.249 & 0.747 & 0.980 & 0.878 & 0.689 & 0.154 \\
\hline
\end{tabular}

Table1. Significance level ( $p<0,05$ in bold) for hypothesis testing under the null hypothesis of no difference between clusters for EEG data.

After splitting DwMT into 3 stages (reading, analysing and selecting options, sketching), still none of the channels shows statistically significant differences between the groups. However, from the topographic heat map that plots the mean log-scale TRP across each cluster of subjects (Figure 3), some interesting activation differences, despite not statistically significant, between the two clusters along the design process emerge. The group with a lower assessment score (second row) had a stronger power demand in the left frontal-central area while reading the contents of the task. Moreover, a reversed hemispherical asymmetry (group A and group B are highly activated in opposite hemispheres) appears across all the stages between the two groups.

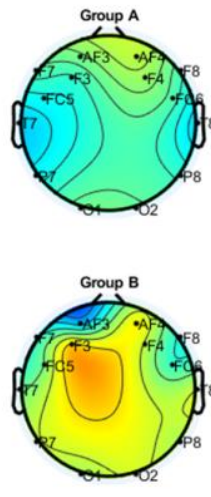

Stage 1
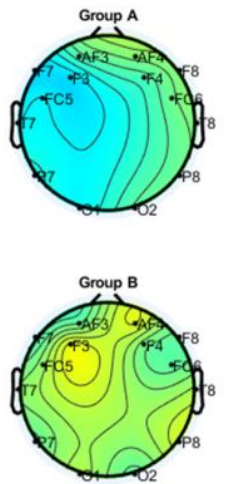

Stage 2
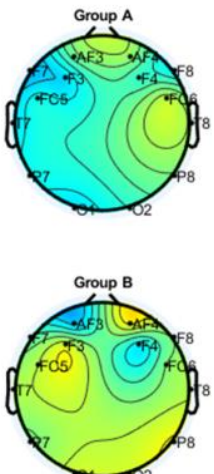

Stage 3

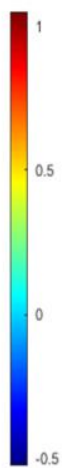

$P<0.05$

Figure 3. TRP for DwMT in the alpha band (7-13Hz). Stage 1 - reading, stage 2 - analysing and making the selection, stage 3 - illustrating. Top row: Group A, Bottom row: Group $B$

\subsubsection{Clustering based on the EEG activation}

Subjects were also clustered according to their brain activation as measured via EEG data. Coherently with section 4.2.1, Group A collects the subjects that have stronger brain activation, group B collects the residuals. Consistently with the analysis of outliers in section 4.2.1, there are no outliers for TTCT, 
5 for PS and 2 for DwMT. Then, group A and B are composed of 10 and 9 subjects for TTCT. For the PS task, group A has 8 subjects and group B 6. Eventually, for the DwMT task, 8 and 9 subjects are respectively in group A and B.

Not surprisingly, this clustering approach boosts the identification of statistically significant differences of EEG activation between groups across multiple channels, with an increasing number along the design stages of task DwMT (Figure 4). However, testing the hypothesis that the two clusters are also different in terms of the scores they received for their task outcomes (null hypothesis of equal distribution between the two clusters) produced results with no statistical significance. However, the score about variety in task TTCT and the degree of EEG activation for the whole task DwMT (Table 2) show a moderate positive correlation between the two ( $\mathrm{rho}=0.4845, \mathrm{p}=0.036)$ and it is worth recalling that both these activities require the subjects to communicate their task outcomes through sketches.

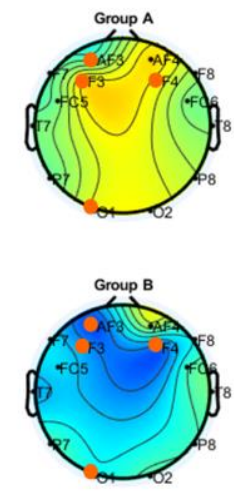

Stage 1
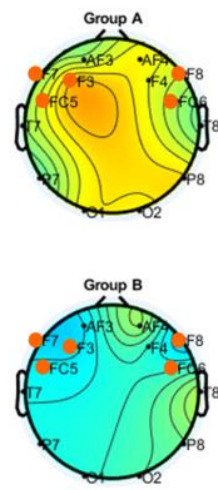

Stage 2
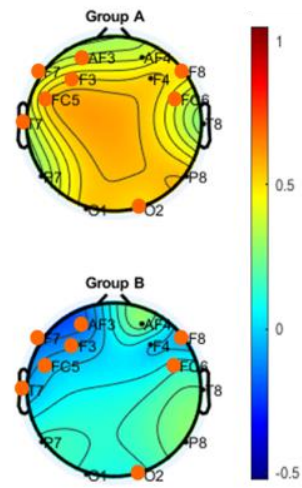

Stage 3

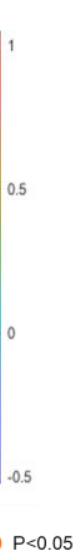

Figure 4. TRP for DwMT in the alpha band (7-13Hz), stage 1 - reading, stage 2 - analysing and making the selection, stage 3 - illustrating.

\begin{tabular}{|l|r|c|c|c|c|c|c|}
\cline { 3 - 8 } \multicolumn{2}{c|}{} & \multicolumn{6}{c|}{ Assessment of the task outcomes } \\
\cline { 3 - 8 } \multicolumn{2}{c|}{} & $\begin{array}{c}\text { TTCT } \\
\text { (variety) }\end{array}$ & $\begin{array}{c}\text { TTCT } \\
\text { (fluency) }\end{array}$ & $\begin{array}{c}\text { DwMT } \\
\text { (clarity) }\end{array}$ & $\begin{array}{c}\text { DwMT } \\
\text { (viability) }\end{array}$ & $\begin{array}{c}\text { PS } \\
\text { (variety) }\end{array}$ & $\begin{array}{c}\text { PS } \\
\text { (fluency) }\end{array}$ \\
\hline \multirow{2}{*}{$\begin{array}{l}\text { EEG } \\
\text { activation }\end{array}$} & TTCT & 0.595 & 0.471 & - & - & - & - \\
\cline { 2 - 8 } & DwMT & $\mathbf{0 . 0 3 6}$ & 0.471 & 0.940 & 0.413 & 0.736 & 0.753 \\
\cline { 2 - 8 } & PS & - & - & - & - & 0.492 & 0.564 \\
\hline
\end{tabular}

Table 2. $p$-values for Pearson's correlation between scores of outcome assessments from each task, and the degree of EEG activation along with each task $(p<0.05$ in bold).

\section{DISCUSSION}

The experimental protocol in the current research project is developed after the experience gained with multiple pilot tests. They enabled exploring the impact of relevant experimental factors, such as the nature of the task for EEG data collection (also with reference to the target subjects' profile); the readability of the text; the performance of the EEG apparatus and the efficiency of the originally developed algorithm for data cleaning. The data processing pipeline for cleaning the artefacts is adopted also in other research projects (Vieira et al., 2020), no excessive artefacts of different perturbing factors remained in the final datasets for running statistical analysis.

From the data collected so far, the experimental protocol and the data processing pipeline proved to be suitable for distinguishing differences between cognitive behaviour based on EEG data. In fact, the number of subjects in each cluster created according to the degree of EEG activation (based on TRP) is generally balanced. Moreover, these clusters show several statistically significant differences across multiple channels. The location of these channels is aligned with previous findings mentioned in the literature, as there is an emergent, despite not statistically significant, the difference between the clusters of subjects for both hemispheres at the frontal and right parietal area (Figure 3).

By assessing the outcome of each task, it is possible to cluster the subjects from the performance point of view. However, the assessment criteria adopted so far, cannot fully cover the different aspects of design. The groups clustered based on the current approach requires further verification. 
When study neurocognition behaviour, the groups for comparison are usually clustered by gender (Razumnikova, 2004), background (Vieira et al., 2020)or year of experience (Fink et al., 2009). On the other hand, clustering subjects according to their EEG activation is a new approach that apparently does not have antecedents in the design literature. The paper checks if the EEG data also explains (or, more simply, is correlated) to the different outcomes rated and scored with traditional approaches. However, with the data collected so far, it was not yet possible to recognize any significant correlation between the EEG activation and the corresponding scores of assessment. That is, the statistical approach returned a non-significant difference between the average score for the outcome of each task once the subjects participating in the experiment get clustered into two groups according to their similar degree of EEG activation. Besides, this lack of statistical significance persists also when more design-related tasks (i.e. that requiring higher-order thinking) like DwMT are not considered as a whole, but in terms of its internal sub-stages (reading, analysing and selecting, sketching), thus changing the clustering into groups and their relationship with the task outcomes.

From this perspective, the correlation between the variety index measured with the outcomes of TTCT and the EEG activation along the stages of task DwMT is promising. In fact, the similarities between these two tasks do not only consist in the way of illustrating the outcome (by sketching). But both require utilizing the given material and making further development. That is, for the TTCT task one should include the two vertical lines in the final sketch. While, for the DwMT task, it is required to combine all the selected feature into a unique idea and visualize the final assembly.

\section{LIMITATIONS AND FUTURE DEVELOPMENT}

Due to the small number of subjects involved because of the COVID pandemics, this analysis just provides preliminary results. A much richer dataset including more subjects for the test is necessary to further validate and reinforce the observed results. All the subjects participating in the experiment have a background in engineering, but with only one year of experience on average. The difference between novices and experts is one of the next targets. Still, because of the lack of subjects, the originality of the ideas could not be used for assessing the outcome of the task TTCT. Nevertheless, the importance of such a factor could not be overlooked in design studies. More experiments are needed to improve the understanding of the correlation between the degree of activation and performance, despite promising results have started to surface. As EEG differences in the design field still suffer from a generic lack of knowledge, it is in the authors' intention to continue the exploration of the correlation between the EEG activation and the outcome of tasks requiring fundamental thinking, as well as the ones with more complex activities that are typical of designing. The already established experimental protocol covers some fundamental thinking tasks, as idea generation, problem-solving, but it also includes a constrained design task that requires higher-level reasoning. By clustering the subjects based on the outcome assessment results, it was possible to distinguish statistically significant differences in the EEG alpha band activation, especially for the channels located on the left frontal and occipital area for the fundamental thinking tasks (Problem solving and TTCT). Further development could include more frequency bands into the analysis to gain a more comprehensive understanding. As no clear difference emerged for EEG activation between the two clusters of subjects when analysing the higher-level design task, a more thorough analysis of the activities the subjects carried out during design with the morphological table is necessary to avoid using just the results of its last stage (sketching). The extension of the experimental campaign with the involvement of additional subjects will also shed light on the potential, yet to be discovered, the correlation between the assessment of activities requiring fundamental thinking (elementary ones) and the degree of EEG activation during constrained design tasks.

\section{REFERENCES}

Abraham, A. (2018), The Neuroscience of Creativity, Cambridge University Press,

Alexiou, K., Zamenopoulos, T. and Gilbert, S. (2011), "Imaging the Designing Brain: A Neurocognitive Exploration of Design Thinking”, Design Computing and Cognition '10, Dordrecht, pp. 489-504,

Appelbaum, L.G., Ales, J.M., Cottereau, B. and Norcia, A.M. (2010), "Configural specificity of the lateral occipital cortex”, Neuropsychologia, Vol. 48 No. 11, pp. 3323-3328,

Arden, R., Chavez, R.S., Grazioplene, R. and Jung, R.E. (2010), "Neuroimaging creativity: A psychometric view”, Behavioural Brain Research, Vol. 214 No. 2, pp. 143-156, 
Benedek, M., Jung, R.E. and Vartanian, O. (2018), “The neural bases of creativity and intelligence: common ground and differences", Neuropsychologia, Vol. 118, pp. 1-3,

Bhattacharya, J. and Petsche, H. (2005), "Drawing on mind's canvas: Differences in cortical integration patterns between artists and non-artists", Human Brain Mapping, Vol. 26 No. 1, pp. 1-14,

Borga, M. and Knutsson, H. (2001), A Canonical Correlation Approach to Blind Source Separation, SE-581 85 Linköping, Sweden.

Chávez, R.A., Graff-Guerrero, A., Garcia-reyna, J., Vaugier, V., et al. (2004), "Neurobiology of creativity: Preliminary results from a brain activation study", Salud Mental, Vol. 27 No. 3, pp. 38-46.

De Clercq, W., Vergult, A., Vanrumste, B., Van Paesschen, W. and Van Huffel, S. (2006), “Canonical Correlation Analysis Applied to Remove Muscle Artifacts From the Electroencephalogram”, IEEE Transactions on Biomedical Engineering, Vol. 53 No. 12, pp. 2583-2587,

Delorme, A. and Makeig, S. (2004), "EEGLAB: An open source toolbox for analysis of single-trial EEG dynamics including independent component analysis", J. Neurosci. Methods, Vol. 134 No. 1, pp. 9-21

Fink, A., Graif, B. and Neubauer, A.C. (2009), "Brain correlates underlying creative thinking: EEG alpha activity in professional vs. novice dancers", NeuroImage, Vol. 46 No. 3, pp. 854-862,

Foxe, J. and Snyder, A. (2011), "The Role of Alpha-Band Brain Oscillations as a Sensory Suppression Mechanism during Selective Attention”, Frontiers in Psychology, Vol. 2, p. 154

Gibson, C., Folley, B.S. and Park, S. (2009), "Enhanced divergent thinking and creativity in musicians: A behavioral and near-infrared spectroscopy study", Brain and Cognition, Vol. 69 No. 1, pp. 162-169,

Göker, M.H. (1997), “The effects of experience during design problem solving”, Design Studies, Vol. 18 No. 4 , pp. 405-426, https://doi.org/10.1016/s0142-694x(97)00009-4.

Guilford, J.P. (1967), The Nature of Human Intelligence, McGraw-Hill, New York.

Heaton, R.K., Chelune, G.J., Talley, J.L., Kay, G.G. and Curtiss, G. (1993), Wisconsin Card Sorting Test Manual: Revised and Expanded, Psychological Assessment Resources Inc, Odessa, FL.

Jackson, A.F. and Bolger, D.J. (2014), “The neurophysiological bases of EEG and EEG measurement: A review for the rest of us", Psychophysiology, Vol. 51 No. 11, pp. 1061-1071

Jiang, X., Bian, G.-B. and Tian, Z. (2019), "Removal of Artifacts from EEG Signals: A Review”, Sensors, Vol. 19 No. 5, https://doi.org/10.3390/s19050987.

Kirchner, W.K. (1958), “Age differences in short-term retention of rapidly changing information”, Journal of Experimental Psychology, Vol. 55 No. 4, pp. 352-358, https://doi.org/10.1037/h0043688.

Klimesch, W., Doppelmayr, M., Schwaiger, J., Auinger, P. and Winkler, T. (1999), "'Paradoxical' alpha synchronization in a memory task", Cognitive Brain Research, Vol. 7 No. 4, pp. 493-501,

Knyazev, G.G. (2007), "Motivation, emotion, and their inhibitory control mirrored in brain oscillations", Neuroscience and Biobehavioral Reviews, Vol. 31 No. 3, pp. 377-395,

Kounios, J. and Beeman, M. (2014), “The Cognitive Neuroscience of Insight”, Annual Review of Psychology, Vol. 65 No. 1, pp. 71-93, https://doi.org/10.1146/annurev-psych-010213-115154.

Martindale, C. and Mines, D. (1975), "Creativity and cortical activation during creative, intellectual and EEG feedback tasks", Biological Psychology, Vol. 3 No. 2, pp. 91-100,

Mednick, S.A. (1968), “The Remote Associates Test”, The Journal of Creative Behavior, Vol. 2 No. 3, pp. 213214, https://doi.org/https://doi.org/10.1002/j.2162-6057.1968.tb00104.x.

Nguyen, T.A. and Zeng, Y. (2010), “Analysis of Design Activities Using EEG Signals”, ASME Design Engineering Technical Conference, Vol. 5, pp. 277-286, https://doi.org/10.1115/DETC2010-28477.

Peirce, J., Gray, J.R., Simpson, S., MacAskill, M., Höchenberger, R., Sogo, H., Kastman, E., et al. (2019), "PsychoPy2: Experiments in behavior made easy", Behavior Research Methods, Vol. 51 No. 1, pp. 195203, https://doi.org/10.3758/s13428-018-01193-y.

Privodnova, E.Y., Volf, N. V. and Knyazev, G.G. (2017), "Specific features of the oscillatory brain activity during the final stage of creative problem solving in young and elderly subjects", Human Physiology, Vol. 43 No. 3, pp. 241-247, https://doi.org/10.1134/S0362119717030161.

Razumnikova, O.M. (2004), "Gender differences in hemispheric organization during divergent thinking: An EEG investigation in human subjects", Neuroscience Letters, Vol. 362 No. 3, pp. 193-195,

Smith, M.E., Gevins, A., Brown, H., Karnik, A. and Du, R. (2001), "Monitoring Task Loading with Multivariate EEG Measures during Complex Forms of Human-Computer Interaction", Human Factors, SAGE Publications Inc, Vol. 43 No. 3, pp. 366-380, https://doi.org/10.1518/001872001775898287.

Torrance, E.P. (1968), Torrance Tests of Creative Thinking, Personnel Press, Princeton, N.J.

Vieira, S., Gero, J.S., Delmoral, J., Li, S., Cascini, G. and Fernandes, A. (2020), "Brain Activity in Constrained and Open Design Spaces: an EEG Study”, ICDC, pp. 068-075, https://doi.org/10.35199/icdc.2020.09.

Vos, D.M., Riès, S., Vanderperren, K., Vanrumste, B., Alario, F.-X., Huffel, V.S. and Burle, B. (2010), "Removal of Muscle Artifacts from EEG Recordings of Spoken Language Production", Neuroinformatics, Vol. 8 No. 2, pp. 135-150, https://doi.org/10.1007/s12021-010-9071-0. 